\title{
Multimodal Imaging of Sarcopenia using Optical Coherence Tomography and Ultrasound in Rat Model
}

\author{
Byeong Hwan Jeon ${ }^{1}$, Yu-Gyeong Chae ${ }^{2,3}$, Sang Seok Hwang ${ }^{2,3}$, Dong Kyu Kim ${ }^{3,4}$, Chulho Oak, \\ Eun-Kee Park ${ }^{3,6 \dagger}$, and Yeh-Chan Ahn ${ }^{2,3} *$ \\ ${ }^{1}$ School of Sports and Health, Kyungsung University, Busan 608-736, Korea \\ ${ }^{2}$ Department of Biomedical Engineering and Center for Marine-Integrated Biomedical Technology, \\ Pukyong National University, Busan 608-737, Korea \\ ${ }^{3}$ Innovative Biomedical Technology Research Center, College of Medicine, Kosin University, \\ Busan 602-702, Korea \\ ${ }^{4}$ Department of Physical Medicine and Rehabilitation, College of Medicine, Kosin University, \\ Busan 602-702, Korea \\ ${ }^{5}$ Department of Internal Medicine, College of Medicine, Kosin University, Busan 602-702, Korea \\ ${ }^{6}$ Department of Medical Humanities and Social Medicine, College of Medicine, Kosin University, \\ Busan 602-702, Korea
}

(Received January 23, 2014 : revised January 30, 2014 : accepted January 31, 2014)

\begin{abstract}
Sarcopenia, or reduced muscle mass and volume, is due to various factors such as senile change, neuronal degeneration, drug, malignancy, and sepsis. Sarcopenia with the aging process has been evidenced by the decline in muscle mass by 0.5 to $1 \%$ per year with $3-5 \%$ reduction in muscle strength for 10 years between the ages of 40 and 50 , and a $1-2 \%$ of decline of mass every year in people aged $60-70$. Therefore, early diagnosis and understanding the mechanism of sarcopenia are crucial in the prevention of muscle loss. However, it is still difficult to image changes of muscle microstructure due to a lack of techniques. In this study, we developed an animal model using denervated rats to induce a rapid atrophy in the tibialis anterior (TA) and imaged its structural changes using optical coherence tomography (OCT) along with histologic and ultrasound analyses. Ultrasound showed changes of overall muscle size. Histology revealed that the atrophic TA muscle displayed an increased size variability of muscle fiber and inflammatory changes. Three dimensional OCT imaged the changes of perimysial grid and muscle fiber structure in real time without sacrifice. These observed advantages of multimodal imaging using OCT and ultrasound would provide clinical benefits in the diagnosis of sarcopenia.
\end{abstract}

Keywords: Sarcopenia, Muscle atrophy, Optical coherence tomography, Ultrasound

OCIS codes : (170.6935) Tissue characterization; (170.3880) Medical and biological imaging; (170.1610) Clinical applications; (170.4500) Optical coherence tomography

\section{INTRODUCTION}

The main roles of skeletal muscle are to cause muscle contraction for physical movement and to maintain body temperature by utilizing by-product heat during contraction. The size and composition of muscle are mainly determined by an individual level of physical activity, nutrients and hormones. However, unavoidable activity limitation due to an injury, illness, genetic disorder, or sedentary lifestyle can cause the reduction of muscle mass, or muscle atrophy [1,2].

According to the previous studies, muscle atrophy occurs by protein turnover which is the imbalance between synthesis and degradation of muscle fiber [3, 4]. During the aging process, muscle loss is caused by various factors; exercise habits, dietary habits and lifestyle, without disease or injury [5]. In general, muscle mass has been shown to decline by 0.5 to $1 \%$ per year with $3-5 \%$ reduction in muscle strength spanning 10 years between the ages of 40 and 50 , and a

\footnotetext{
*Corresponding authors: *ahny@pknu.ac.kr, ${ }^{\dagger}$ ekpark@kosin.ac.kr

Color versions of one or more of the figures in this paper are available online.
} 
$1-2 \%$ of decline of mass every year in people aged $60-70$. Particularly, femoral muscle mass, which has shown significantly greater atrophy in comparison to other muscles, decreased by approximately $24-27 \%$ through the ages of 20 to $70[6,7]$.

Sarcopenia, or reduced muscle mass and volume, is accompanied by the loss of muscle strength because muscle produces strength and power in proportional to cross sectional area of the muscle bundle. This vicious cycle hampers locomotion, increases the risks of injuries and diseases, limits independent living, and collectively deteriorates the quality of life [8-10].

Maintaining or increasing muscle tone through physical activity or exercise, reverses the muscle degeneracy via cellular control reaction to up-regulate the synthesis of contractile muscle fiber as well as to rebuild the associated tissues including connective, nervous, and vascular tissues. As physical function is recovered, the risk of chronic diseases and injuries can be lowered $[2,10]$.

Clinicians not only need to understand the mechanism of sarcopenia but confirming an early diagnosis would be crucial in the prevention of muscle loss. However, it is made difficult by a lack of techniques to image changes of muscle structure. Histology is the gold standard to provide a morphological information but it requires an excision of muscle tissue. Therefore, in vivo imaging modalities such as ultrasound, computed tomography, or magnetic resonance imaging have been used to image muscle. Clinical ultrasound has a low spatial resolution $(>100 \mu \mathrm{m})$ not enough to image individual muscle fibers, and it experiences artifacts which are dependent on sonographer's skill and the system itself. Computed tomography induces a tissue ionization that limits the practice of routine checks due to safety precautions. Magnetic resonance imaging is too expensive to be used for muscle diagnosis.

Optical coherence tomography (OCT) is usually based on Michelson interferometer utilizing backscattering of near infra-red light from tissue. It provides cross-sectional images of micro-tissue morphology with a high resolution $(2-10 \mu \mathrm{m})$ in a non-invasive manner but imaging depth is limited to 1-2 mm. Previous OCT studies [11-13] have visualized changes of muscle microstructure using an $m d x$ mouse model for Duchenne muscular dystrophy. In order to image deep skeletal muscle structure, a needle probe has been proposed [14]. However, no OCT study has been done to image changes in muscle structure associated with sarcopenia, to the best of our knowledge. In this study, we developed an animal model using denervated rats to induce rapid muscle atrophy and imaged muscle structural changes using OCT along with histological and ultrasound analyses.

\section{MATERIALS AND METHODS}

\subsection{Animals and Denervation}

Two male Sprague-Dowley rats $(200 \sim 250 \mathrm{~g})$ were kept

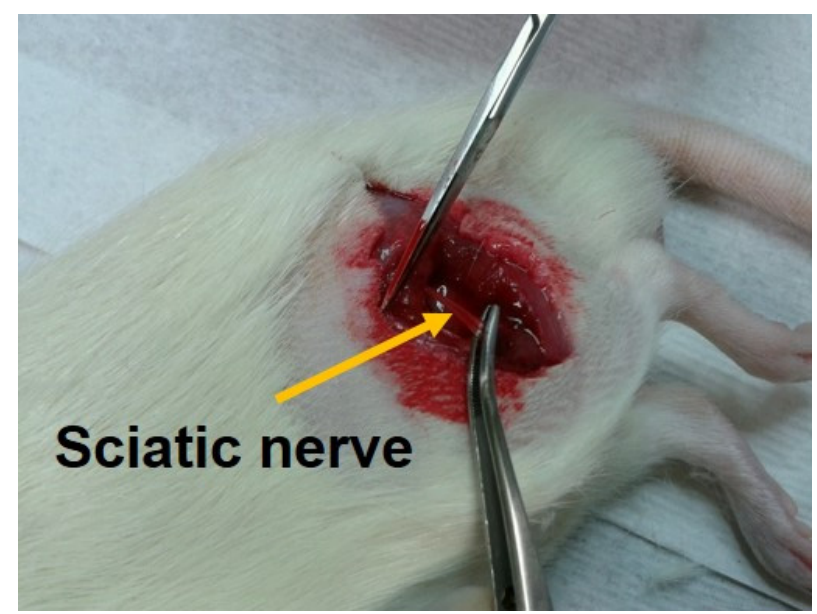

FIG. 1. Left sciatic nerve was dissected by surgical procedure to make a rat model for sarcopenia.

in a controlled environment of $23 \pm 2^{\circ} \mathrm{C}, 55 \pm 5 \%$ humidity and a 12-hour light, 12-hour dark lighting cycle. Commercial rodent pellet diet and water were supplied ad libitum. All animal procedures were conducted in accordance with the guidelines published in the Guide for the Care and Use of Laboratory Animals (DHEW publication NIH 85-23, revised 2010, Office of Science and Health Reports, DRR/NIH, Bethesda, MD). The study protocol was approved by the Committee on Animal Research of the College of Medicine at Kosin University.

To induce rapid atrophy of muscles in laboratory rats, sciatic nerves were dissected in accordance with surgical procedure, as previously described [15]. In brief, the sciatic nerve of the hind limb was dissected and removed $(5-10 \mathrm{~mm})$ unilaterally and the counter part was sham-operated as a control.

Before surgery, rats were anaesthetized using an intraperitoneal injection of pentobarbital sodium $(50 \mathrm{mg} / \mathrm{kg} \mathrm{BW})$. The left sciatic nerve was separated and removed (4-5 $\mathrm{mm})$ as shown in Fig. 1.

A rapid loss of muscle mass was observed in 2 weeks after denervation and progressively reduced during the following 2 weeks [16]. Four weeks after the surgery tibialis anterior (TA) muscles were separated from both distinguished hind limbs of each animal.

\subsection{Histopathology}

The rat was euthanized using $\mathrm{CO}_{2}$ gas. TA muscle was dissected and fixed by $10 \%$ neutral buffered formalin (NBF), and embedded in paraffin. Four-micrometer-thick serial sections were stained with hematoxylin and eosin (H\&E) and examined by microscopy.

\subsection{Optical Coherence Tomography System}

We constructed a spectrometer-based OCT system as shown in Fig. 2. It was a fiber optic Michelson interferometer. A broadband SLED light source (Broadlighter D855, Superlum, Ireland) with a center wavelength of $850 \mathrm{~nm}$ and a full width 


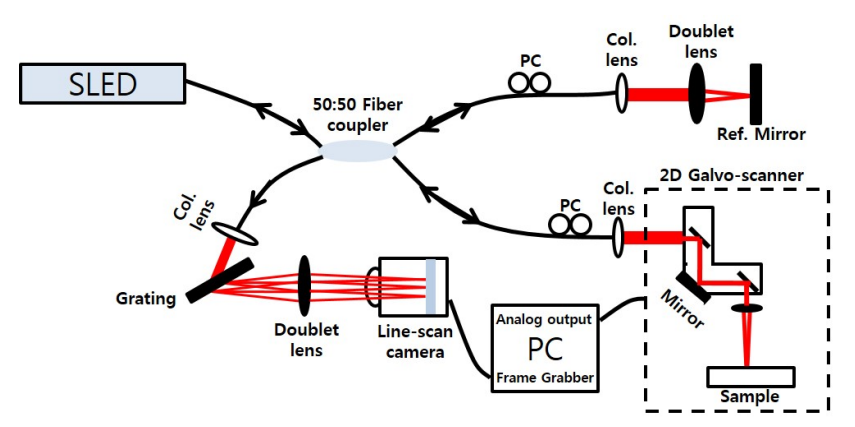

FIG. 2. Schematic diagram of the optical coherence tomography system developed in this study.

at half maximum of $100 \mathrm{~nm}$ was used. The light was directed to a 50:50 coupler that split the light into reference and sample arms. Backscattered lights from a reference mirror and a sample produced an interference fringe. The fringe pattern was collected by a line scan camera (Sprint spL4096-140km, Basler, PA, USA) with a line rate of 140 $\mathrm{kHz}$ and 4096 pixels. A two-axis scanner was customized using two galvanometers $(6220 \mathrm{H}$, Cambridge Technology, MA, USA). Five hundred twelve B-mode images of each TA muscle were acquired at 10 fps for 1024 lateral pixels. Three dimensional images $\left(5.12 \times 2.56 \times 3.2 \mathrm{~mm}^{3}\right)$ were rendered using the 512 B-mode images. A cross-sectional image could be reconstructed in any direction by image processing. The point spread function was measured and showed a depth resolution of $4 \mu \mathrm{m}$ in air, a roll-off of $12 \mathrm{~dB} / \mathrm{mm}$, and a signal-to-noise ratio of $103 \mathrm{~dB}$.

\subsection{High Frequency Ultrasound System}

As a multimodal imaging approach, we also used a high resolution ultrasound system (Sonix Touch, Ultrasonix, Canada) with a high frequency linear transducer (40-8 $\mathrm{MHz}$ ) with a depth range of $0.2-3 \mathrm{~cm}$ and a geometric focus of $6 \mathrm{~mm}$. Imaging parameters for this study were set as follows: $40 \mathrm{MHz}$ of frequency, $2 \mathrm{~cm}$ of imaging depth, $1.2 \mathrm{~cm}$ of imaging width, $85 \mathrm{~dB}$ of dynamic range, and 63 $\mathrm{Hz}$ of frame rate.

\section{RESULTS}

Figure 3(a) shows a typical in vivo ultrasound image (transverse view) of a healthy TA muscle. TA muscle was identified by being imaged with tibia and fibula. Similarly, ultrasound provided overall muscle size that was measured along long and short axes in both healthy and atrophic TA muscles. Ultrasound indicated an apparent reduction of muscle size in both axes of the atrophic TA muscle compared with the healthy one (Data is not shown in this article) as previous studies had obtained [17]. When comparing histologic images of the healthy and atrophic TA muscles (Figs. 3(b), (c)), there was a significant difference in size, showing a good correspondence with ultrasonographic

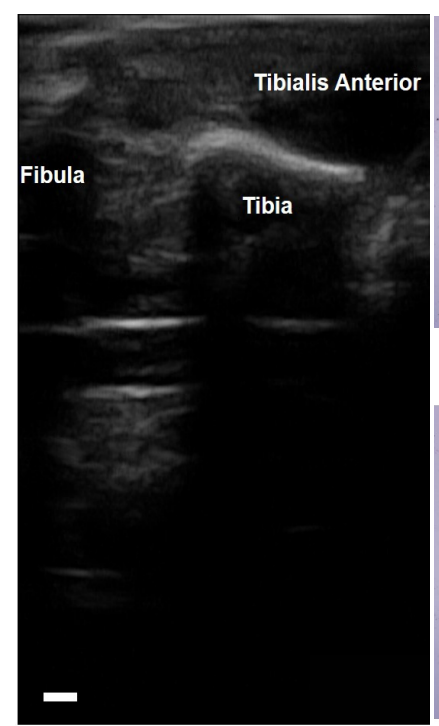

(a)

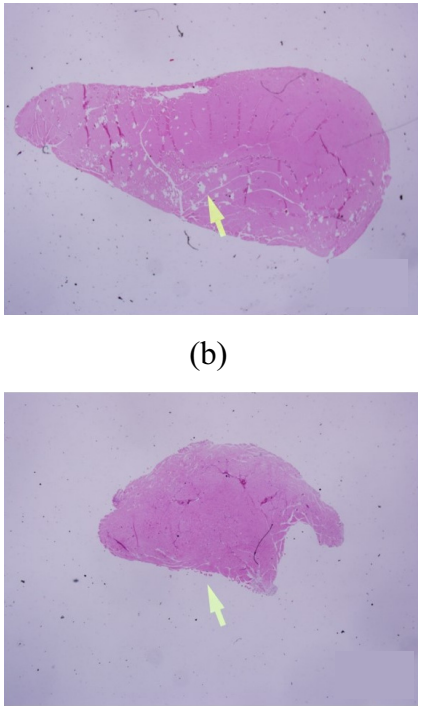

(c)
FIG. 3. In vivo high resolution (40 MHz) ultrasound image of healthy tibialis anterior (TA) of rat (a), histopathology of healthy (b) and atrophic (c) TA muscles. Scale bar for ultrasound and histologic images: $1 \mathrm{~mm}$.

findings. Ultrasound, however, failed to show detailed structures or structural changes of TA muscles.

OCT was applied to image detailed structures of TA muscles. Figures 4(a) and (b) are in vivo cross-sectional images (transverse view) of the healthy and atrophic TA muscles, respectively. There was a thicker subcutaneous fat layer over atrophic TA muscle than over a healthy one as shown in Figs. 4(a) and (b). Due to the thick fat layer, atrophic muscle was almost out of imaging depth (Fig. $4 b)$. In order to observe atrophic TA muscle, the fat layer was removed after sacrifice. Figure 4(c) is an ex vivo OCT image of atrophic TA muscle.

OCT images of Figs. 4(a) and (c) clearly shows perimysium that was confirmed by the histologic images (Figs. 4d,e). The perimysium is a connective tissue that wraps bundles of muscle fibers also known as fascicles. The atrophic muscle (Fig. 4c) showed significant reduction in the size of fascicles and, therefore, a fine grid of perimysium in comparison with the healthy one. The size of muscle fibers was also finer and the size variability of muscle fibers was higher for the atrophic TA muscle than for the healthy one, as was verified by the histologic images. Since OCT images of Figs. 4(a) and (c) were cross-sectional images of muscle fibers, muscle fibers were imaged as white spots. A high density of white spots in the grid of perimysium characterized the atrophic TA muscle. Another difference between the atrophic and the healthy TA muscles is the thickness of perimysium (Figs. $4 a, c)$. The perimysium of atrophic muscle was thicker and this architectural difference was consistent with findings from the histologic staining with $\mathrm{H} \& \mathrm{E}$, where numerous 


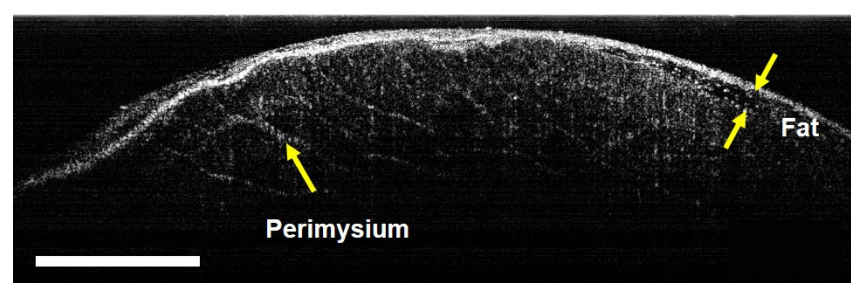

(a)

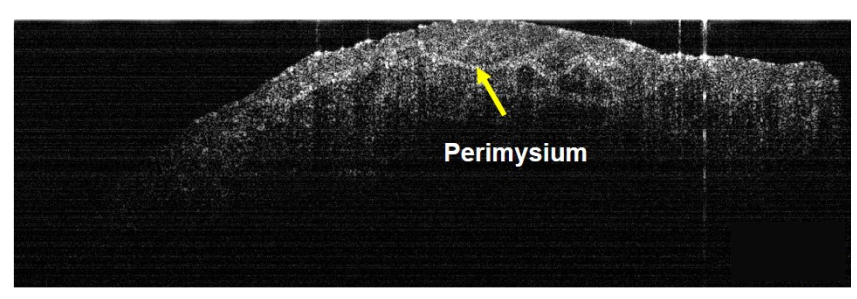

(c)

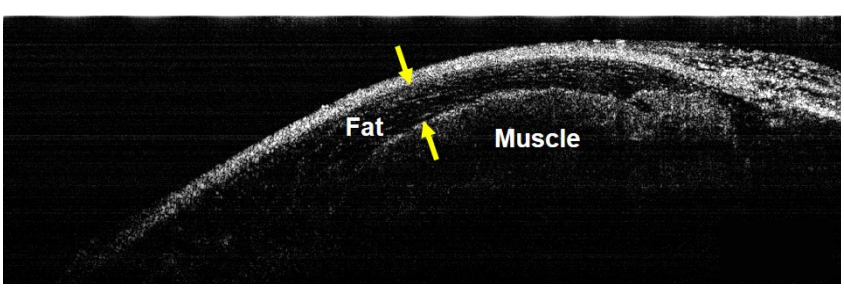

(b)
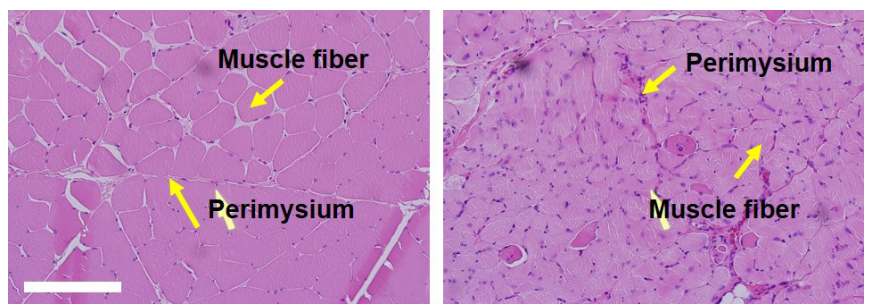

(d)

FIG. 4. In vivo OCT images of healthy (a) and atrophic (b) tibialis anterior (TA) muscles. Fat layer thickness in the atrophic TA muscle is thicker than that of the healthy one, as indicated by the arrows. Ex vivo OCT image (c) of atrophic TA muscle without the fat layer. Histopathology of healthy (a) and atrophic (b) TA muscles. Scale bar for OCT: $1 \mathrm{~mm}$, scale bar for histology: $100 \mu \mathrm{m}$.

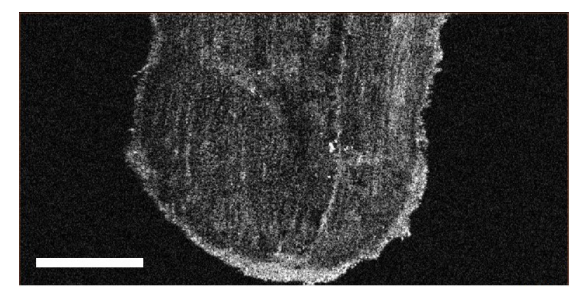

(a)

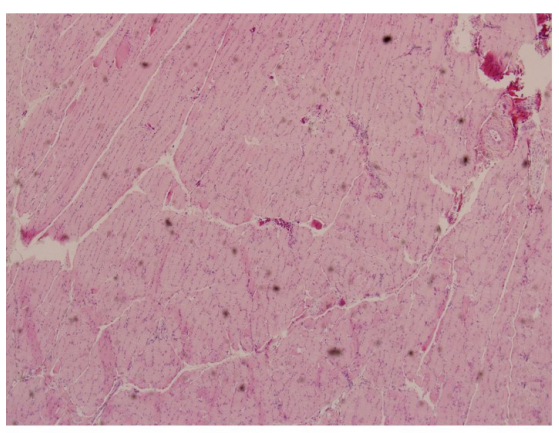

(c)

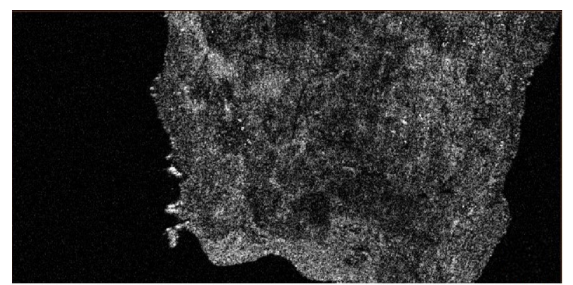

(b)

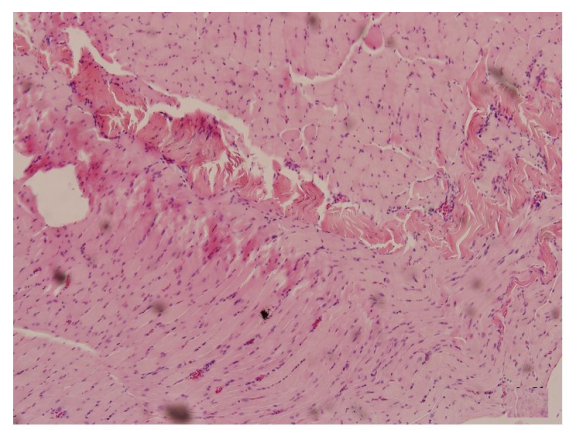

(d)

FIG. 5. En face OCT images of healthy (a) and atrophic (b) tibialis anterior muscles. En face histologic images of healthy (c) and atrophic (d) tibialis anterior muscles. Scale bar for OCT and histology: $1 \mathrm{~mm}$.

inflammatory cells were infiltrated in perimysial spaces as shown in Fig. 4(e).

En face images were reconstructed from 3D-OCT data sets using the protocol described in the Materials and Methods section. Figure 5 depicts representative en face OCT images at a depth of $0.4 \mathrm{~mm}$ and corresponding H\&E stained histologic images. Figures 5(a) and (c) are images of healthy TA muscle. The pattern of longitudinal structures of muscle fibers, as shown in Fig. 5(c) existed throughout all en face OCT images with different depths, with a shape and a size similar to those expected for healthy muscle fibers. The healthy fibers generally appeared more uniform in texture in a coarse grid of perimysium. In the atrophic TA muscle (Figs. 5(b), (d)), however, the fiber pattern was irregular throughout different depths and the perimysial grid was fine.

\section{DISCUSSION}

The present study evaluated a multimodal platform of OCT and ultrasound to compare structures of healthy and atrophic TA muscles and demonstrated a complementary 
benefit of multimodal imaging in the most suitable rat model for sarcopenia. OCT, combined with pathology, showed an advantage in visualization of muscle microstructure over other imaging modalities such as ultrasound. Histology revealed that the atrophic TA muscle displayed an increased size variability of muscle fiber and inflammatory changes. OCT could image the changes of the perimysial grid and muscle fiber structure in real time without sacrifice.

Ultrasound has been widely used for diagnosis of musculoskeletal disease in clinical settings. However, there are few studies using ultrasound with a high resolution for muscular disease. In this study, ultrasound illustrated overall muscle size with distinct anatomical relationship between the healthy and atrophic TA muscles. Even with a high frequency transducer (40-8 MHz), ultrasound demonstrated a limitation in showing microstructures in muscle, especially in small animals such as rats. Therefore, the potential advantage of multimodal imaging in muscle degeneration using OCT and ultrasound may be of great importance. These are potential benefits in its application for diagnosing clinical muscular degeneration.

In clinical settings, there are various factors affecting sarcopenia such as senile change, neuronal degeneration, drug, malignancy, and sepsis. Therefore, the multimodal platform using OCT and ultrasound for evaluation of sarcopenia may be useful for many future applications.

\section{CONCLUSION}

Our results from this study employing OCT illustrated that this method could be a potential tool for monitoring the muscle atrophy induced by denervation. Morphological changes were visualized by OCT with supportive images taken from H\&E staining and ultrasound analysis. Ultrasound provided a reduction of overall muscle size which OCT was not able to image due to a limited imaging depth. Structural changes observed by OCT may be attributed to the changes in the size, orientation, and regularity of microarchitecture within the muscle, including changes in perimysium and bundles of muscle fibers (fascicles). Our observation reinforces the potential for the use of OCT and ultrasound as a minimally-invasive diagnostic tool for sarcopenia.

\section{ACKNOWLEDGMENT}

This study was supported by Kyungsung University Research Grants in 2013.

\section{REFERENCES}

1. A. E. Emery, "The muscular dystrophies," Lancet 359, 687695 (2002).

2. S. B. Chargé and M. A. Rudnicki, "Cellular and molecular regulation of muscle regeneration," Physiol. Rev. 84, 209-
238 (2004).

3. W. J. Evans, "Skeletal muscle loss: Cachexia, sarcopenia, and inactivity," Am. J. Clin. Nutr. 91, 1123S-1127S (2010).

4. M. Sandri, "Signaling in muscle atrophy and hypertrophy," Physiology (Bethesda) 23, 160-170 (2008).

5. V. A. Hughes, W. R. Frontera, M. Wood, W. J. Evans, G. E. Dallal, R. Roubenoff, and M. A. F. Singh, "Longitudinal muscle strength changes in older adults: influence of muscle mass, physical activity, and health," J. Gerontol. A. Biol. Sci. Med. Sci. 56, B209-17 (2001).

6. M. V. Narici and N. Maffulli, "Sarcopenia: Characteristics, mechanisms and functional significance," Br. Med. Bull. 95, 139-159 (2010).

7. J. Ward, "Sarcopenia and sarcopenic obesity: Is it time the health system accepted fitness of older people as a health responsibility?," Australas. J. Ageing 30, 61-62 (2011).

8. W. R. Frontera, V. A. Hughes, R. A. Fielding, M. A. Fiatarone, W. J. Evans, and R. Roubenoff, "Aging of skeletal muscle: a 12-yr longitudinal study," J. Appl. Physiol. 88, 1321-1326 (2000).

9. T. Rantanen, R. Sakari-Rantala, and E. Heikkinen, "Muscle strength before and mortality after a bone fracture in older people," Scand. J. Med. Sci. Sports 12, 296-300 (2002).

10. F. Dela and M. Kjaer, "Resistance training, insulin sensitivity and muscle function in the elderly," Essays Biochem. 42, 75-88 (2006).

11. B. R. Klyen, J. J. Armstrong, S. G. Adie, H. G. Radley, M. D. Grounds, and D. D. Sampson, "Three-dimensional optical coherence tomography of whole-muscle autografts as a precursor to morphological assessment of muscular dystrophy in mice," J. Biomed. Opt. 13, 011003 (2008).

12. B. R. Klyen, T. Shavlakadze, H. G. Radley-Crabb, M. D. Grounds, and D. D. Sampson, "Identification of muscle necrosis in the $m d x$ mouse model of Duchenne muscular dystrophy using three-dimensional optical coherence tomography," J. Biomed. Opt. 16, 076013 (2011).

13. R. M. Lovering, S. B. Shah, S. J. P. Pratt, W. Gong, and Y. Chen, "Architecture of healthy and dystrophic muscles detected by optical coherence tomography," Muscle Nerve 47, 588-590 (2013).

14. X. Yang, D. Lorenser, R. A. McLaughlin, R. W. Kirk, M. Edmond, M. C. Simpson, M. D. Grounds, and D. D. Sampson, "Imaging deep skeletal muscle structure using a high-sensitivity ultrathin side-viewing optical coherence tomography needle probe," Biomed. Opt. Express 5, 136148 (2014).

15. J. P. Hyatt, R. R. Roy, K. M. Baldwin, and V. R. Edgerton, "Nerve activity-independent regulation of skeletal muscle atrophy: Role of MyoD and myogenin in satellite cells and myonuclei," Am. J. Physiol. Cell Physiol. 285, C1161-C1173 (2003).

16. J. M. Sacheck, J. P. Hyatt, A. Raffaello, R. T. Jagoe, R. R. Roy, V. R. Edgerton, S. H. Lecker, and A. L. Goldberg, "Rapid disuse and denervation atrophy involve transcriptional changes similar to those of muscle wasting during systemic diseases," FASEB J. 21, 140-155 (2007).

17. D. Mayans, M. S. Cartwright, and F. O. Walker, "Neuromuscular ultrasonography: Quantifying muscle and nerve measurements," Phys. Med. Rehabil. Clin. N. Am. 23, 133148 (2012). 